\title{
Idiopathic neonatal atrial flutter
}

INSERM

\section{Source}

INSERM. (1999). Orphanet: an online rare disease and orphan drug data base. Idiopathic neonatal atrial flutter. ORPHA:45452

Idiopathic neonatal atrial flutter (AFL) is a rare rhythm disorder, characterized by sustained tachycardia in newborns and infants with an atrial rate often at around 440 beats/minute (range 340-580). AFL may manifest as asymptomatic tachycardia, congestive heart failure or hydrops. 